\title{
VESICAL METASTASIS OF GASTRIC ADENOCARCINOMA
}

\author{
ALBERTO A. ANTUNES, TIBÉRIO M. SIQUEIRA JR, EVANDRO FALCÃO
}

Service of Urology, Getúlio Vargas Hospital, Recife, Pernambuco, Brazil

\begin{abstract}
Metastatic vesical tumors are rare, and constitute approximately $1 \%$ of all neoplasias affecting this organ. The authors report the case of a 63-year old woman with vesical metastasis of gastric adenocarcinoma.

Patient presented signs of cachexia and complained of left lumbar pain and dysuria unresponsive to antibiotic therapy for approximately 5 months. She reported a previous partial gastrectomy due to ulcerative undifferentiated gastric adenocarcinoma 1 year and 9 months before.

Cystoscopy revealed an extensive vegetative lesion in bladder, occupying its entire mucosal surface. The biopsy revealed metastatic signet-ring cell adenocarcinoma.
\end{abstract}

Key words: bladder; metastasis; gastric cancer

Int Braz J Urol. 2004; 30: 403-5

\section{INTRODUCTION}

In Brazil, bladder cancer is the fourth more frequent neoplasia in men, and the second most prevalent urogenital neoplasia. Bladder tumors can be primary or metastatic. Metastatic tumors are rare, and constitute approximately $1 \%$ of all neoplasias affecting this organ (1).

Signs and symptoms resulting from secondary vesical involvement are present in approximately $20 \%$ of cases and the majority of information relative to such cases derives from autopsy studies (2).

The authors report the case of a 63-year old woman with vesical metastasis of gastric adenocarcinoma.

\section{CASE REPORT}

FMS, 63 years old, complained of left lumbar pain and dysuria unresponsive to antibiotic therapy for approximately 5 months. She also reported weight loss that was not quantified during this period.
Physical examination revealed enlarged and ascitic abdomen, without palpable masses, and painful at lower abdomen. She reported a previous partial gastrectomy 1 year and 9 months before, due to ulcerative undifferentiated gastric adenocarcinoma that reached the organ's serosa.

Serum creatinine on presentation was $3.5 \mathrm{mg} /$ dL. Urine culture was negative. She underwent a cystoscopy that revealed an extensive vegetative lesion in bladder, practically occupying the entire mucosal surface. There were no necrotic areas or signs of urinary infection. A biopsy of the vesical lesion was performed.

Abdominal computerized tomography evidenced bilateral hydronephrosis and voluminous ascites (Figure-1). Bladder presented thickened wall, suggesting tumoral process (Figure-2). Chest x-ray normal. The pathological analysis of the biopsy specimen revealed metastatic signet-ring cell adenocarcinoma (Figure-3).

Patient refused adjuvant treatment and was discharged from hospital on the 10th hospitalization 


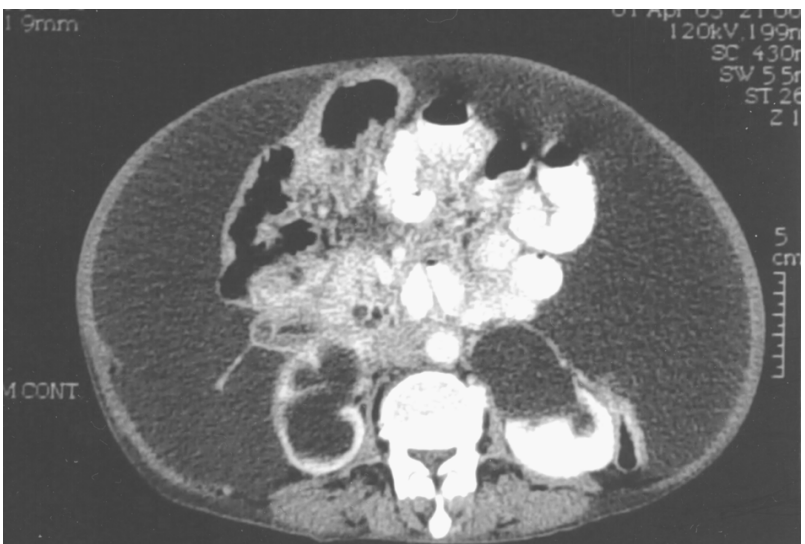

Figure 1 - Abdominal computerized tomography showing bilateral hydronephrosis and voluminous ascites.

day with symptomatic medication and conservative treatment. She presented stable condition after an 8month follow-up.

\section{COMMENTS}

Vesical metastases of gastric carcinoma correspond to approximately $1 \%$ of all neoplasias affecting bladder and, in $31 \%$ of cases, the primary focus is stomach (1).

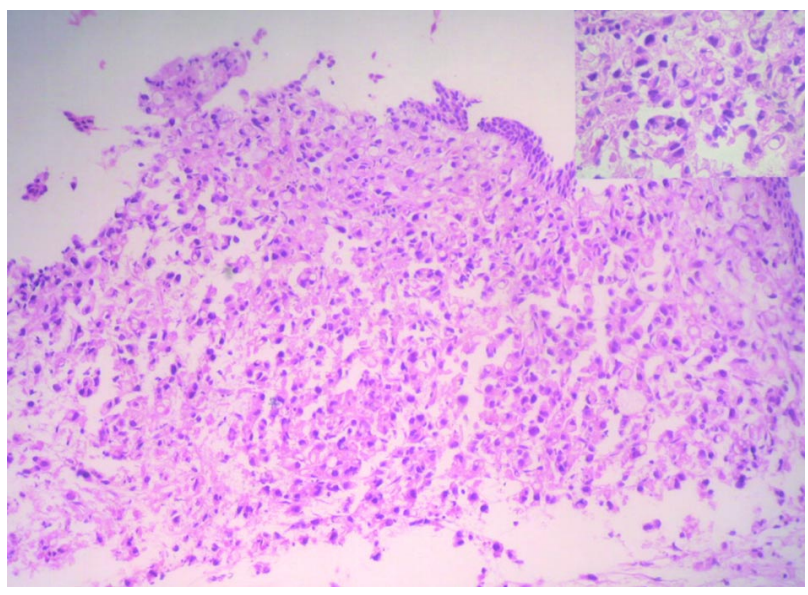

Figure 3 - Photomicrography showing infiltration of signet-ring cell adenocarcinoma in bladder wall (HE, X250), detail with magnification X400.

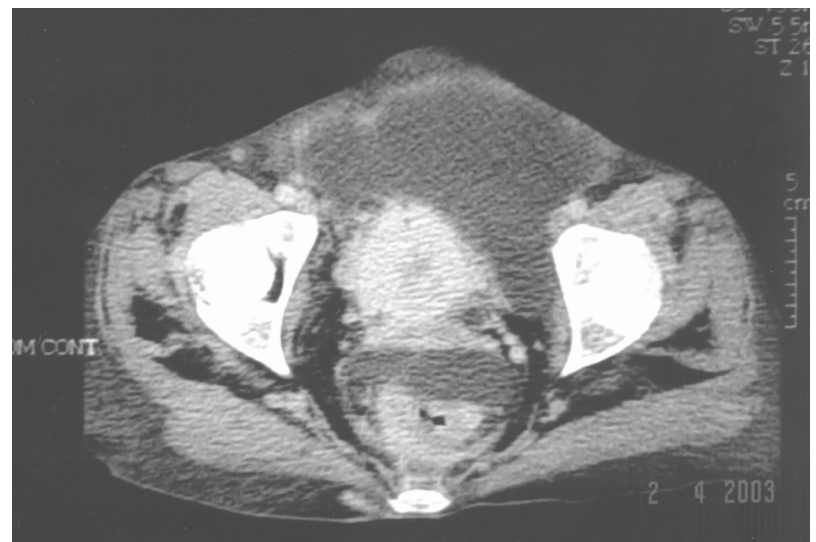

Figure 2 - Pelvic computerized tomography showing thickened bladder wall, suggesting tumoral infiltration.

Among cases of metastatic vesical tumors published to the moment, the incidence is similar between genders, and mean age ranges from 44 to 63 years (3).

Potential mechanisms that can contribute for the development of secondary bladder lesions are direct extension of the primary focus, implant of exfoliated cells from ureter and renal pelvis, and lymphatic, hematogenic or peritoneal dissemination from a distant focus (2).

In our patient, urinary symptoms motivated the performance of complementary exams such as cystoscopy and abdominal computerized tomography, which resulted in the diagnosis of metastatic bladder tumor. Generally speaking, treatment with curative intention is not possible due to the metastatic characteristic of the disease, and adjuvant chemotherapy is indicated in some cases, though with unsatisfactory results.

After diagnosing a bladder adenocarcinoma, we must keep in mind the possibility of primary stomach or colon lesions, since in a few cases this can be the first clinical manifestation in these patients.

\section{REFERENCES}

1. Valero Puerta JA, Medina Perez M, Garcia Carriazo M, Valpuesta Fernandez I, Sanchez Gonzalez M, et 
al.: Bladder metastasis of signet-ring cell adenocarcinoma from the stomach. Arch Esp Urol. 2000; 53: 83941.

2. Leddy FF, Peterson NE, Ning TC: Urogenital linitis plastica metastatic from stomach. Urology. 1992; 39: 464-7.
3. Saba NF, Hoening DM, Cohen SI: Metastatic signetring cell adenocarcinoma to the urinary bladder. Acta Oncol. 1997; 36: 219-20.

Received: February 16, 2004 Accepted after revision: July 19, 2004

\footnotetext{
Correspondence address:

Alberto Azoubel Antunes

R. Três de Maio, 17 / 31

04044-020, São Paulo, SP

E-mail: betoazoubel@yahoo.com.br
} 